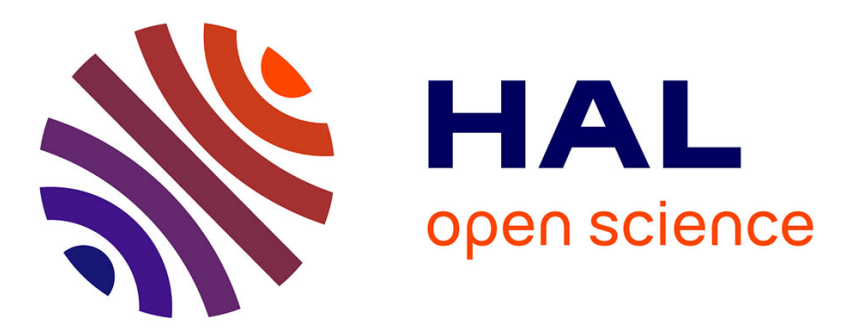

\title{
Ionization of phenol by single electron impact: triple differential cross sections
}

Lena Mouawad, Paul-Antoine Hervieux, Claude Dal Cappello, Ziad El Bitar

\section{To cite this version:}

Lena Mouawad, Paul-Antoine Hervieux, Claude Dal Cappello, Ziad El Bitar. Ionization of phenol by single electron impact: triple differential cross sections. J.Phys.B, 2020, 53 (2), pp.025202. 10.1088/1361-6455/ab5be6 . hal-02431437

\section{HAL Id: hal-02431437 \\ https://hal.science/hal-02431437}

Submitted on 24 Nov 2020

HAL is a multi-disciplinary open access archive for the deposit and dissemination of scientific research documents, whether they are published or not. The documents may come from teaching and research institutions in France or abroad, or from public or private research centers.
L'archive ouverte pluridisciplinaire HAL, est destinée au dépôt et à la diffusion de documents scientifiques de niveau recherche, publiés ou non, émanant des établissements d'enseignement et de recherche français ou étrangers, des laboratoires publics ou privés. 


\title{
Ionization of phenol by single electron impact: Triple differential cross sections
}

\author{
Lena Mouawad ${ }^{1}$, Paul-Antoine Hervieux ${ }^{1}$, Claude Dal Cappello², Ziad El Bitar ${ }^{3}$ \\ ${ }^{1}$ Université de Strasbourg, CNRS, IPCMS UMR 7504, F-67000 Strasbourg, France \\ ${ }^{2}$ Université de Lorraine, CNRS, LPCT UMR 7019 , F-57000 Metz, France \\ ${ }^{3}$ Université de Strasbourg, CNRS, IPHC UMR 7178, F-67000 Strasbourg, France
}

\begin{abstract}
Triple differential cross sections (TDCS) for the ionization by single electron impact of the highest and next-highest occupied molecular orbitals of phenol are provided in this work. A first Born approach is used, describing the ejected electron by a distorted wave. The molecular wave functions are developed in a single-center form using the computational chemistry program Gaussian. The TDCSs are calculated for an unknown molecular orientation with the proper average (PA) approach and using four different models. In the distorted wave model (DW) the atoms positions do not intervene in the interaction potential between the incoming electron and the molecular target while in the distorted wave multicenter model (DW-MC) they are taken into account. In the DW-Gamow and the DW-MC-Gamow models, the repulsion between the outgoing electrons is described by adding the Gamow factor to the DW and DW-MC models. In general, all results are in good agreement with the experimental data. A small difference is observed between the DW and DW-MC results in the recoil region. Adding the Gamow factor improves the angular position and the relative amplitude of the two peaks observed in the binary region. When the TDCSs are compared to other theoretical results relying on the orientation averaged molecular orbital (OAMO) approximation, the improved agreement with the experimental data reinforces the importance of the proper average approach.
\end{abstract}

\section{Introduction}

Electron interactions with molecules have gained a lot of interest in the recent past years due to their importance in many research fields, especially in radiation therapy $[1,2]$. In cancer treatment using ionizing radiation, the primary particles produce large quantities of secondary electrons which interact with the biological molecules. The ionization of these molecules by electron impact plays a major role in inducing damage to cancerous cells especially by the resulting low energy electrons $[3,4]$. Hence, extensive research has been conducted in the aim of providing accurate cross sections for the electron impact ionization of biomolecules [5-10].

A complete description of the ionization dynamics is ensured by calculating the triple differential cross sections (TDCS) which involve all kinematic parameters. This quantity can be measured in (e,2e) experiments where the ejected and scattered electrons coming from the same ionization event are detected in coincidence and their energy and direction are identified. These are difficult experiments especially for complex targets for which the preparation of the sample in gaseous phase is not always evident. Moreover, the geometry of the molecule and its orientation and even in some cases the ionized orbitals cannot be precisely pinpointed.

Testing a theoretical model with the experimental data is hence very delicate. This is especially the case for biological molecules. For example, in the (e,2e) experiment for tetrahydrofuran, a structural unit of the DNA phosphate backbone which can exist as three different isomers, it was not possible to trace back the electrons to the ionization of a single THF isomer $[11,12]$. It was therefore necessary to calculate the TDCSs for the two most populated isomers and compare the total TDCS to the experimental TDCSs [9]. Another example is the pyrimidine experiment [13]. In this experiment, the energy resolution being higher than the binding energy separation between the targeted orbital and the following two orbitals, the detected electrons resulting from the ionization of these three orbitals could not be distinguished. This means that, for a valid comparison between theory and experiment, total theoretical TDCSs from the three orbitals should be compared to the experimental TDCSs [10]. These considerations not only add to the computing time but also make it harder to accurately judge the validity of the theory since the exact contribution of each orbital or isomer to the final result is unknown. Not to mention the uncertainties of the experiments that can sometimes be so large that it becomes irrelevant to compare the exact theoretical calculations to the measurements that cover a wide range of relative amplitudes, knowing that experimental TDCSs are very rarely provided in absolute units and are most often in relative units. Hence only the overall shape of the TDCS can be compared to the experimental TDCS pattern. 
The unknown molecular orientation in the provided experimental TDCS leads to one of the biggest challenges of theoretical studies. In (e,2e) experiments, it is still not possible to identify the exact orientation of the molecule at the time it was ionized. So the experimental TDCSs are always provided for a random orientation of the molecule. To account for this in theory, the TDCSs should be averaged over all possible molecular orientations using the proper average method (PA) $[7,9,14,15]$. This is done by calculating the TDCS for different orientations of the molecule and then comparing the average TDCS to the experimental TDCS. One can imagine how time consuming this would be especially for complex molecules like most biological molecules. For that reason, another approach was suggested, the orientation average molecular orbital (OAMO) approximation [16], which consists in performing this average on the molecular wave functions before calculating a single TDCS for an average-orientation molecular orbital.

The OAMO approximation was used alongside the molecular three-body distorted wave (M3DW) model to calculate TDCSs for relatively complex targets such as $\mathrm{CH}_{2} \mathrm{O}_{2}$ (formic acid) [17], $\mathrm{C}_{6} \mathrm{H}_{4} \mathrm{O}_{2}$ (parabenzoquinone) [18], $\mathrm{C}_{4} \mathrm{H}_{4} \mathrm{~N}_{2}$ (pyrimidine) [13] and $\mathrm{C}_{5} \mathrm{H}_{4} \mathrm{O}_{2}$ (furfural) [19]. In the M3DW model, three distorted waves are used to describe the incident, scattered and ejected electrons. The interaction between the outgoing electrons is directly included in the transition matrix through a Ward-Macek average Coulomb-distortion factor [16, 20], as in the BBK method used for the hydrogen atom [21]. Hence, the post collision interactions (PCI) are included to all orders of perturbation theory in M3DW. Despite the strength of this model, the M3DW-OAMO TDCSs were not in agreement with the experiments in general especially for orbitals with dominant $p$-character. The OAMO was also used for simpler molecules such as $\mathrm{H}_{2}$ [22], $\mathrm{N}_{2}$ [23], $\mathrm{CH}_{4}$ [24], and the comparison between PA and OAMO TDCSs for some molecules [25, 26] shows that the OAMO method is not always valid and is merely an approximation that was judged as necessary because of the computing limitations that do not allow the use of PA for complex targets.

The only models that were used for more complex molecules either rely on the OAMO approximation, or on other approximations to reduce the heaviness of computing. One of these methods is the multi-center distorted wave (MCDW) model where non-diagonal terms in the potential matrix are ignored to make the calculations possible [27-30]. Another approximation is to calculate the TDCS as a weighted sum of atomic TDCSs corresponding to the atoms of the molecule, in the completely neglected differential overlap (CNDO) description, which fails in reproducing the experimental TDCS for many molecules $[7,31]$. To our knowledge, the methodology that we propose is the only one that allows the calculation of the TDCSs for complex targets without having to make such drastic approximations. In previous work, we have demonstrated that with our FBA approach, the DW TDCSs are in reasonable agreement with experimental data for formic acid [8], tetrahydrofuran [9] and pyrimidine [10] using the PA method. In this work, we show that good results are also obtained for phenol, and we also upgrade the DW model to three new models.

Phenol $\left(\mathrm{C}_{6} \mathrm{H}_{5} \mathrm{OH}\right)$ is an organic molecule that consists of a hydroxyl group (-OH) attached to a carbon atom in a hydrocarbon ring (figure 1). It is used in medicine as a precursor of pharmaceutical drugs and in the treatment of inflammatory disease [32]. It also intervenes in the extraction of DNA from cell culture samples in molecular biology [33]. Many studies were interested in investigating electron interactions with phenol [34-37].

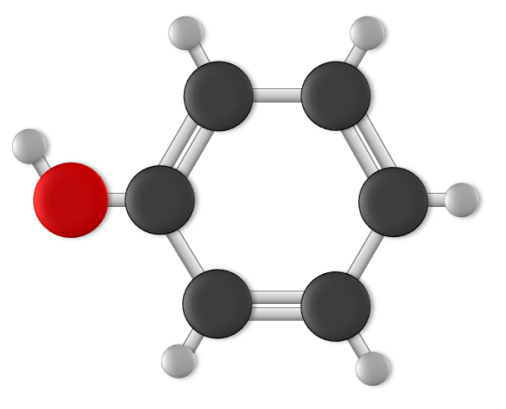

Figure 1: Phenol molecule: $\mathrm{C}_{6} \mathrm{H}_{5} \mathrm{OH}$.

Twenty-five occupied molecular orbitals are identified for phenol. A diagrammatic representation of the highest and next-highest occupied molecular orbitals, $4 \mathrm{a}^{\prime \prime}$ and $3 \mathrm{a}^{\prime \prime}$ respectively, are shown in figure 2 . The ionization energies of these two orbitals are $8.58 \mathrm{eV}$ and $9.41 \mathrm{eV}[38]$. 

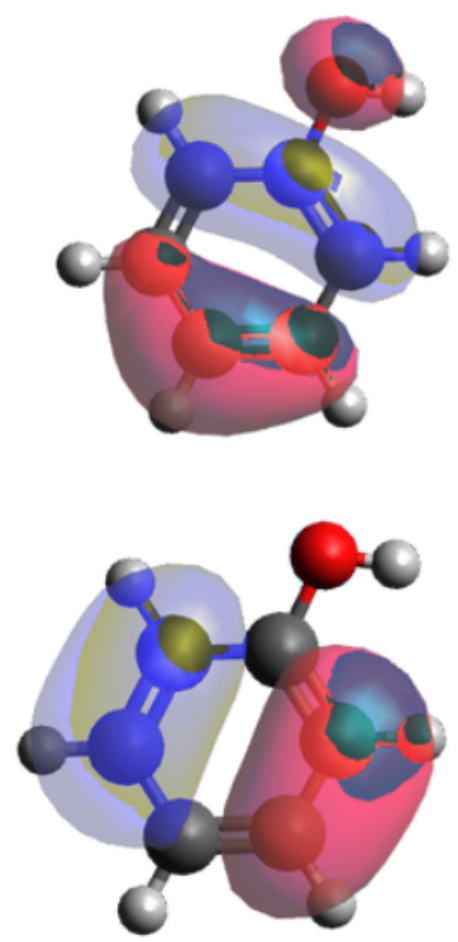

Figure 2: HOMO (top) and $\mathrm{HOMO}^{-1}$ (bottom) of phenol.

We compute multi-center molecular wave functions for these two orbitals using Gaussian [39]. After expressing these wave functions in a single-center formalism [5, 40,41], they are used alongside the incident and scattered plane wave functions and the ejected electron's distorted wave function to calculate the transition amplitude. The post collision interaction of the molecule's electrons and the ejected electron is taken into account using an average isotropic distorted potential in the DW method. The atoms positions are included in the potential seen by the incident electron in the DWMC model. In these two models, the interaction between the ejected and scattered electrons is not taken into account. By multiplying the TDCSs from each of these models by the Gamow factor [20], the repulsion between the outgoing electrons is included within the DW-Gamow and DW-MC-Gamow models.

At low computational cost, we compute the TDCSs for the ionization of $4 \mathrm{a}^{\prime \prime}$ and $3 \mathrm{a}^{\prime \prime}$ in the kinematics of the (e,2e) experiment that was performed in the intermediate energy regime and in a coplanar asymmetric geometry [38]. The total TDCSs from these two orbitals and calculated using the DW, DW-MC, DW-Gamow and DW-MC-Gamow methods are compared to the experimental data and to M3DW-OAMO TDCSs from [38]. The influence of using a multi-center/singlecenter potential and including the final state electron-electron interaction is hence investigated by comparing the four TDCSs. The results show that our methodology based on the PA method succeeds in general in describing the ionization of phenol while in some kinematics the M3DW-OAMO method clearly fails.

In the second section we describe the theoretical models that are used in this work before moving on to presenting the results in the third section. We finally conclude on our work in the last section. Unless specified otherwise, atomic units are used.

\section{Theoretical framework}

The ionization of phenol by single electron impact may be represented by

$$
\mathrm{e}_{i}\left(E_{i}, \overrightarrow{k_{i}}\right)+\mathrm{C}_{6} \mathrm{H}_{5} \mathrm{OH} \rightarrow \mathrm{C}_{6} \mathrm{H}_{5} \mathrm{OH}^{+}+\mathrm{e}_{s}\left(E_{s}, \overrightarrow{k_{s}}\right)+\mathrm{e}_{e}\left(E_{e}, \overrightarrow{k_{e}}\right)
$$

where $E_{i}, E_{s}, E_{e}$ and $\overrightarrow{k_{i}}, \overrightarrow{k_{s}}, \overrightarrow{k_{e}}$ are the energies and momenta of the incident, scattered and ejected electrons, respectively.

For a particular molecular orientation defined by Euler angles $(\alpha, \beta, \gamma)$, the triple differential cross section (TDCS) for this reaction reads:

$$
\sigma_{\alpha, \beta, \gamma}^{(4)}=\frac{\mathrm{d}^{4} \sigma}{\mathrm{d} \Omega_{s} \mathrm{~d} \Omega_{e} \mathrm{~d} E_{e} \mathrm{~d} \Omega_{\alpha \beta \gamma}},
$$


where $\Omega_{s}$ and $\Omega_{e}$ are the scattered and ejected electron solid angles respectively and $\mathrm{d} \Omega_{\alpha \beta \gamma}=\mathrm{d} \alpha \sin \beta \mathrm{d} \beta \mathrm{d} \gamma$.

It is calculated through the transition amplitude $T_{\mathrm{B}_{1}}$ from the initial state $\Psi_{i}$ to the final state $\Psi_{f}$ as follows:

$$
\sigma_{\alpha, \beta, \gamma}^{(4)}=\frac{2}{(2 \pi)^{5}} \frac{k_{s} k_{e}}{k_{i}}\left|T_{\mathrm{B}_{1}}\right|^{2}
$$

In the first Born approximation (FBA), the transition amplitude is given by:

$$
T_{\mathrm{B}_{1}}=\left\langle\Psi_{f}|V| \Psi_{i}\right\rangle
$$

where $V$ is the interaction potential between the incident electron and the target:

$$
V=\sum_{j=1}^{N_{\mathrm{e}}} \frac{1}{\left|\vec{R}-\vec{r}_{j}\right|}-\sum_{n=1}^{N_{\mathrm{at}}} \frac{Z_{n}}{\left|\vec{R}-\vec{R}_{n}\right|} .
$$

In the equation above, $N_{\mathrm{e}}$ and $N_{\mathrm{at}}$ are the number of bound electrons and the number of atoms in the molecule respectively, $\vec{R}, \vec{r}_{j}$ and $\vec{R}_{n}$ are the position vectors of the incident electron, the $j^{\text {th }}$ molecular electron and the $n^{\text {th }}$ atom respectively, and $Z_{n}$ is the atomic number of the $n^{\text {th }}$ atom.

The initial state wave function $\Psi_{i}$ is the product of the incident electron plane wave function $e^{i \vec{k}_{i} \cdot \vec{R}}$ and the molecular target's wave function $\Psi_{\text {target }}$ :

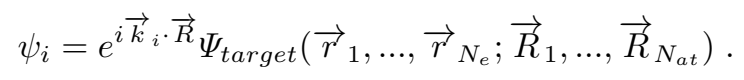

The final state wave function $\Psi_{f}$ is the product of the scattered electron plane wave function $e^{i \vec{k}_{s} \cdot \vec{R}}$, the ejected electron distorted wave function $\mathscr{F}_{\vec{k}_{e}}^{(-)}\left(\vec{r}_{1}\right)$ and the residual ion wave function $\Psi_{i o n}$ :

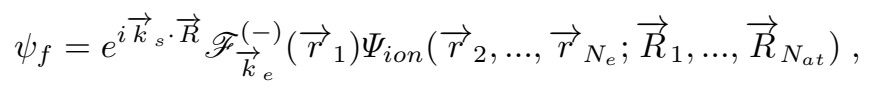

where $\vec{r}_{1}$ is the position vector of the ejected electron.

Using Bethe equality and reducing the $N_{e}+1$ electrons problem to a one active electron problem, the transition amplitude becomes:

$$
T_{\mathrm{B}_{1}}=\frac{4 \pi}{k^{2}}\left\langle\mathscr{F}_{\vec{k}_{e}}^{(-)}\left(\vec{r}_{1}\right)\left|e^{i \vec{k} \cdot \vec{r}_{1}}-\frac{1}{N_{\mathrm{e}}} \sum_{n=1}^{N_{\mathrm{at}}} Z_{n} e^{i \vec{k}^{2} \cdot \vec{R}_{n}}\right| \phi_{i}\left(\vec{r}_{1}\right)\right\rangle,
$$

with $\vec{k}=\vec{k}_{i}-\vec{k}_{s}$ the momentum transfer and $\phi_{i}\left(\vec{r}_{1}\right)$ the initial-state wave function of the active electron. Consequently to orientation averaging, integrating over the atomic orientations $d \hat{R}_{n}$ leads to:

$$
T_{\mathrm{B}_{1}}=\frac{4 \pi}{k^{2}}\left\langle\underset{\mathscr{F}_{e}}{(-)}\left(\vec{r}_{1}\right)\left|e^{i \vec{k}^{\prime} \cdot \vec{r}_{1}}-\frac{1}{N_{e}} \sum_{n=1}^{N_{\mathrm{at}}} Z_{n} \frac{\sin \left(k R_{n}\right)}{k R_{n}}\right| \phi_{i}\left(\vec{r}_{1}\right)\right\rangle .
$$

In the DW model, we assume that the momentum transfer from the projectile to the molecule is so small that the sum over the number of atoms tends to 1 and the transition amplitude is taken as:

$$
T_{\mathrm{B}_{1}}=\frac{4 \pi}{k^{2}}\left\langle\underset{\mathscr{F}_{k}}{(-)}\left(\vec{r}_{1}\right)\left|e^{i \vec{k} \cdot \vec{r}_{1}}-1\right| \phi_{i}\left(\vec{r}_{1}\right)\right\rangle .
$$

In the multi-center method (DW-MC), we do not make this approximation and rather calculate the sum directly in equation (9) by taking into account the position of the different atoms making up the molecule.

Replacing in (3), the TDCS becomes:

$$
\sigma_{\alpha, \beta, \gamma}^{(4)}=\frac{2}{(2 \pi)^{5}} \frac{k_{s} k_{e}}{k_{i}}\left|\frac{4 \pi}{k^{2}} \int d \vec{r}_{1} \mathscr{F}_{\vec{k}_{e}}^{(-)^{*}}\left(\vec{r}_{1}\right) \phi_{i}\left(\vec{r}_{1}\right)\left[e^{i \vec{k}^{*} \cdot \vec{r}_{1}}-\Lambda\right]\right|^{2}
$$

with $\int d \vec{r}_{1}=\int r_{1}^{2} d r_{1} \int d \hat{r}_{1}$, where $r_{1}$ and $\hat{r}_{1}$ are respectively the radial and angular components of $\vec{r}_{1}$, and taking $\Lambda=1$ in the DW model and $\Lambda=\sum_{n=1}^{N_{a t}} \frac{Z_{n}}{N_{e}} \frac{\sin \left(k R_{n}\right)}{k R_{n}}$ in the DW-MC model. 
In both models the ejected electron distorted wave function is:

$$
\mathscr{F}_{\overrightarrow{k_{e}}}^{(-)}(\vec{r})=\sum_{l_{e}, m_{e}}(4 \pi) i^{l_{e}} e^{-i \delta_{l_{e}}} \frac{F_{l_{e}}\left(k_{e} r\right)}{k_{e} r} Y_{l_{e}, m_{e}}^{*}\left(\hat{k}_{e}\right) Y_{l_{e}, m_{e}}(\hat{r})
$$

where $\delta_{l_{e}}$ is the phase shift and the radial wave function $F_{l_{e}}\left(k_{e} r\right)$ is a solution of the following differential equation with an angular averaged distortion potential $U_{i}(r)$ :

$$
\begin{gathered}
{\left[\frac{1}{2} \frac{\mathrm{d}^{2}}{\mathrm{~d} r^{2}}+E_{e}-\frac{l_{e}\left(l_{e}+1\right)}{2 r^{2}}-U_{i}(r)\right] F_{l_{e}}\left(k_{e} r\right)=0,} \\
U_{i}(r)=\frac{1}{4 \pi} \int\left[\sum_{j=1}^{N_{a t}} \frac{Z_{j}}{\left|\vec{r}-\vec{R}_{j}\right|}+\sum_{j=1}^{N_{o r b}}\left(2-\delta_{i j}\right) \int \frac{\left|\phi_{j}\left(\vec{r}_{j}\right)\right|^{2}}{\left|\vec{r}-\vec{r}_{j}\right|} \mathrm{d} \overrightarrow{r_{j}}\right] \mathrm{d} \hat{r},
\end{gathered}
$$

assuming that the electron is ejected from the $i^{\text {th }}$ orbital, with $N_{\text {orb }}$ the number of molecular orbitals, $\delta_{i j}$ the Kronecker delta, and $\phi_{j}\left(\overrightarrow{r_{j}}\right)$ the wave function of the bound electron in the $j^{t h}$ orbital.

The molecular wave functions $\phi_{j}$ are generated using Gaussian [39] with the 6-31G basis set at the Restricted HartreeFock (RHF) level of theory as single-center wave functions [5-7, 40, 41] under the form:

$$
\phi_{j}(\vec{r})=\sum_{\lambda, m_{\lambda}} \tilde{R}_{\lambda, m_{\lambda}}^{j}(r) Y_{\lambda, m_{\lambda}}(\hat{r})
$$

Finally, the TDCS is calculated for an average molecular orientation by averaging the orientation dependent TDCSs over Euler angles within the proper average method [6-10]:

$$
\sigma^{(3)}=\frac{\mathrm{d}^{3} \sigma}{\mathrm{d} \Omega_{s} \mathrm{~d} \Omega_{e} \mathrm{~d} E_{e}}=\frac{1}{8 \pi^{2}} \int \sigma_{\alpha, \beta, \gamma}^{(4)} \mathrm{d} \Omega_{\alpha \beta \gamma} .
$$

Assuming that the electron is ejected from the $i^{\text {th }}$ orbital, the TDCS reads:

$$
\sigma^{(3), i}=\frac{k_{s}}{k_{e} k_{i}} \frac{64}{k^{4}} \sum_{\lambda, m_{\lambda}} \sum_{\mu} \frac{1}{\hat{\lambda}}\left|\mathcal{S}_{\lambda, m_{\lambda}, \mu}^{i}\right|^{2},
$$

with

$$
\begin{gathered}
\mathcal{S}_{\lambda, m_{\lambda}, \mu}^{i}=\sum_{l_{e}, m_{e}} \sum_{l}(-1)^{\mu} i^{\left(l-l_{e}\right)} e^{i \delta_{l_{e}}} Y_{l_{e}, m_{e}}\left(\hat{k}_{e}\right) Y_{l, \mu-m_{e}}(\hat{k}) \mathcal{R}_{l_{e}, l, \lambda, m_{\lambda}}^{i} \mathcal{A}_{l_{e}, m_{e}, l}^{\lambda, \mu}, \\
\mathcal{R}_{l_{e}, l, \lambda, m_{\lambda}}^{i}=\int_{0}^{\infty} r^{2} \tilde{R}_{\lambda, m_{\lambda}}^{i}(r) \frac{F_{l_{e}}\left(k_{e} r\right)}{r}\left(j_{l}(k r)-\Lambda\right) d r \\
\mathcal{A}_{l_{e}, m_{e}, l}^{\lambda, \mu}=\left(\hat{l}_{e} \hat{l} \hat{\lambda}\right)^{\frac{1}{2}}\left(\begin{array}{ccc}
l_{e} & l & \lambda \\
0 & 0 & 0
\end{array}\right)\left(\begin{array}{ccc}
l_{e} & l & \lambda \\
-m_{e} & m_{e}-\mu & \mu
\end{array}\right),
\end{gathered}
$$

where $j_{l}(k r)$ is the spherical Bessel function and $\hat{l_{e}}, \hat{l}, \hat{\lambda}$ are defined as $\hat{l}=2 l+1$.

In the DW-Gamow and DW-MC-Gamow models, we introduce the Gamow factor $N_{e e}[20]$ in order to include the interaction between the scattered and ejected electrons:

$$
N_{e e}=\frac{\pi / k_{e e}}{e^{\pi / k_{e e}}-1}
$$

where $k_{e e}=\mu v_{e e} ; \mu=1 / 2$ being the reduced mass for the two electrons and $v_{e e}=\left|\vec{k}_{e}-\vec{k}_{s}\right|$ the relative velocity between them.

By calculating the Gamow factor at each ejected electon angle and multiplying the TDCS with this factor, the TDCS has a more realistic description of the repulsion between the outgoing electrons. 


\section{Results and discussion}

The (e,2e) experiment providing relative TDCSs for phenol was performed by Da Silva and coworkers [38] in intermediate energy kinematics. For an electron beam energy of $250 \mathrm{eV}$, the analyzer detecting the scattered electron was fixed at three different angles $\theta_{s}=-5^{\circ},-10^{\circ},-15^{\circ}$ and the ejected electron analyzer was rotated along a range of angles in the forward and backward directions of the momentum transfer detecting ejected electrons with an energy of $20 \mathrm{eV}$. The incident energy resolution was estimated to $1.1 \mathrm{eV} \mathrm{FWHM,} \mathrm{it} \mathrm{is} \mathrm{larger} \mathrm{than} \mathrm{the} \mathrm{binding} \mathrm{energy} \mathrm{difference} \mathrm{of} \mathrm{the} \mathrm{HOMO}$ and $\mathrm{HOMO}^{-1}$. Hence the detected electrons result from the ionization of both orbitals.

We begin by investigating the effect of the multi-center potential by comparing the TDCSs obtained with the DW and DW-MC methods. We recall that the difference between the two models is mathematically represented by the $\Lambda$ factor:

$$
\Lambda=\sum_{n=1}^{13} \frac{Z_{n}}{50} \frac{\sin \left(k R_{n}\right)}{k R_{n}},
$$

which is fully included in the DW-MC model and taken as equal to 1 in the DW model. Taking into account the position of the atoms in the considered geometry of the phenol molecule, we calculate this factor for different values of the momentum transfer $k$ (figure 3). The three experimental kinematic conditions [38] correspond to $k=0.44$ a.u. when the scattered angle is fixed at $-5^{\circ}, k=0.77$ a.u. when the scattered angle is fixed at $-10^{\circ}$ and $k=1.12$ a.u. when the scattered angle is fixed at $-15^{\circ}$. The three cases are indicated by the vertical dashed lines in figure 3 from left to right respectively. Evidently, the $\Lambda$ factor is overestimated by the DW model $(\Lambda=1)$ in the three cases.

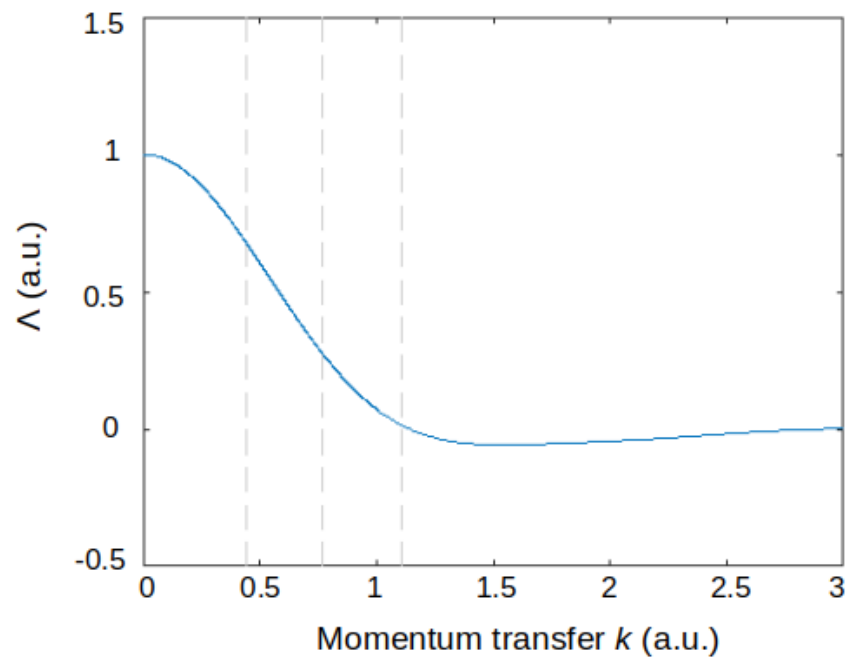

Figure 3: The $\Lambda$ factor (solid line) defined in equation (22) calculated for different values of the momentum transfer $k$ from the incident electron to a phenol molecule in the considered geometry. The vertical dashed lines delimit the values of momentum transfer that correspond to the three kinematic conditions that will be treated in this section; $k=0.44,0.77$ and 1.12 a.u. from left to right respectively.

Figure 3 shows that, for very small magnitudes of the momentum transfer $(k<0.1$ a.u. $)$, the $\Lambda$ factor is very close to 1 . It then decreases as the momentum transfer $k$ increases and reaches zero when $k=1.12 \mathrm{a}$.u. for $\theta_{s}=-15^{\circ}$. This case corresponds to the bound Bethe ridge where the momentum transfer $k$ and the ejected electron momentum $k_{e}$ are equal. Consequently, we expect to have more difference between the DW and DW-MC TDCSs as the momentum transfer $k$ increases.

The theoretical models presented in the previous section are used to calculate the TDCSs for the ionization of the HOMO $\left(4 \mathrm{a}^{\prime \prime}\right)$ and $\mathrm{HOMO}^{-1}\left(3 \mathrm{a}^{\prime \prime}\right)$ of phenol and the total TDCS is compared to the experimental data from [38]. Before comparing the two models, we show the individual contributions of the $4 \mathrm{a}^{\prime \prime}$ (solid line) and $3 \mathrm{a}^{\prime \prime}$ (dash-dotted line) orbitals to the total TDCS (dashed line) in figure 4. We only show DW results in this figure but the behavior of the two contributions is very similar using the DW-MC method. 


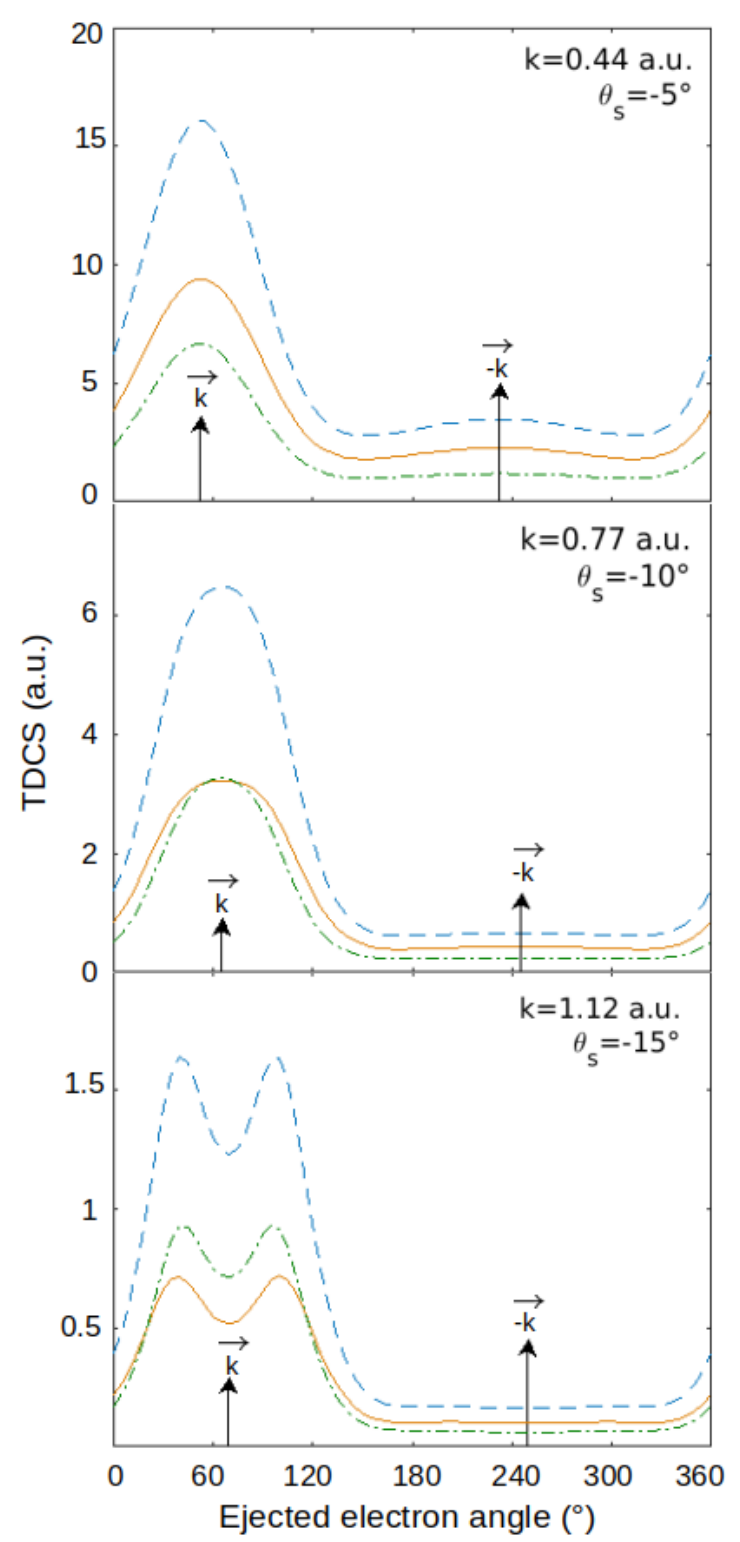

Figure 4: Triple differential cross sections for the ionization of the HOMO $\left(4 \mathrm{a}^{\prime \prime}\right)$ and $\operatorname{HOMO}^{-1}\left(3 \mathrm{a}^{\prime \prime}\right)$ of phenol using the DW model. Solid lines: $4 \mathrm{a}^{\prime \prime}$, dash-dotted lines: $3 \mathrm{a}^{\prime \prime}$, dashed lines: $4 \mathrm{a}^{\prime \prime}+3 \mathrm{a}^{\prime \prime}$. The kinematic conditions are: $E_{i}=250 \mathrm{eV}, E_{e}=20 \mathrm{eV}$, $\theta_{s}=-5^{\circ},-10^{\circ},-15^{\circ}$ in the upper, middle and lower graph respectively. All the TDCSs are in atomic units.

The TDCS from both orbitals exhibits the same shape in the three kinematics: a single binary peak that becomes wider and splits into two binary peaks as the momentum transfer increases. This similar behavior is due to the common nature of these two orbitals which have a dominant $p$-character. It results that the total TDCS also has the same shape and is less sensitive to the approximative attribution of contributions from each of the two orbitals.

Assuming that the TDCS of the two orbitals equally contribute to the total TDCS, we compare the total TDCSs with the DW and the DW-MC methods in figure 5 by the dashed and solid lines respectively. As expected, the difference between the two results is more pronounced with the increase of the momentum transfer from the top to the low panel. 


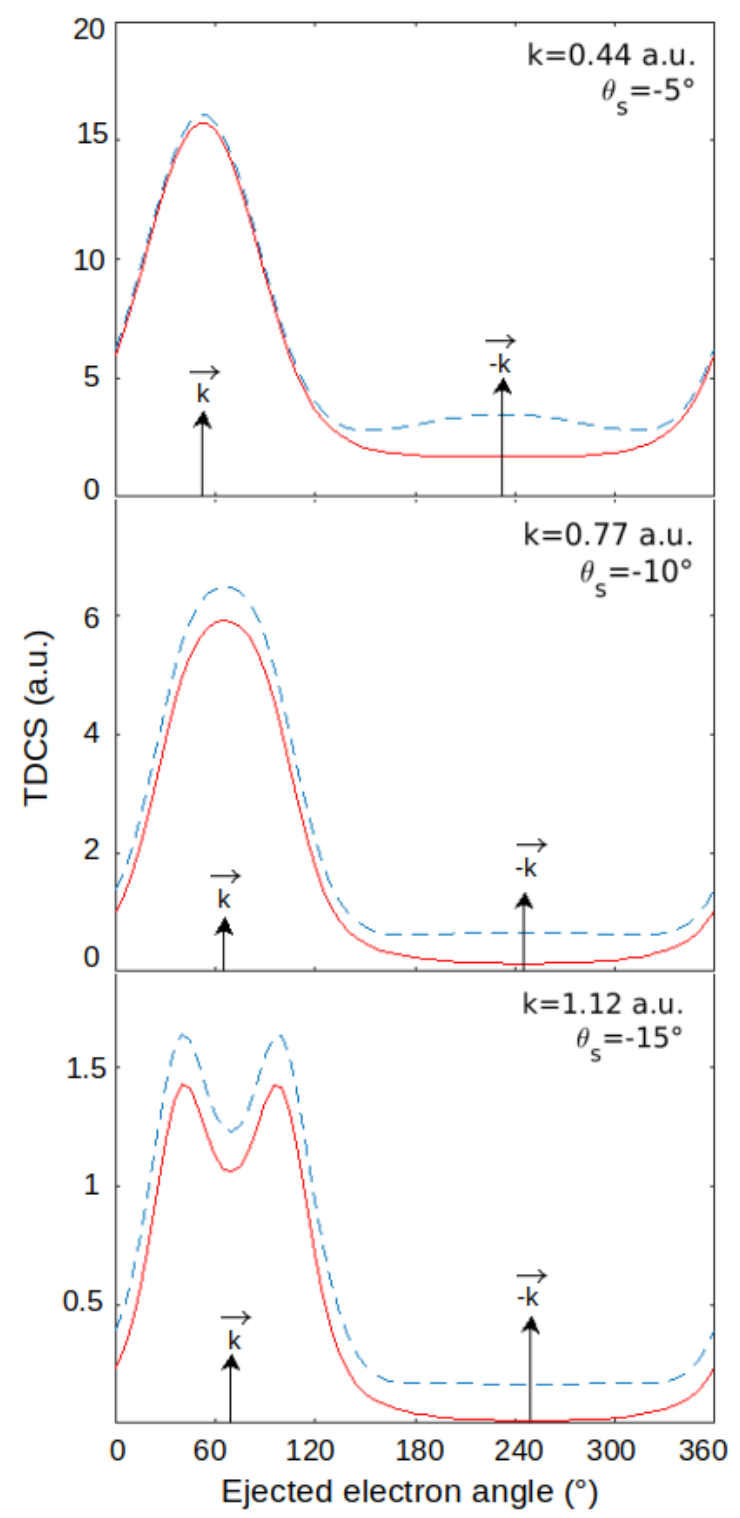

Figure 5: Total triple differential cross sections for the ionization of the HOMO $\left(4 \mathrm{a}^{\prime \prime}\right)$ and $\mathrm{HOMO}^{-1}\left(3 \mathrm{a}^{\prime \prime}\right)$ of phenol using the DW (dashed lines) and DW-MC (solid lines) models. The kinematic conditions are: $E_{i}=250 \mathrm{eV}, E_{e}=20 \mathrm{eV}, \theta_{s}=-5^{\circ},-10^{\circ},-15^{\circ}$ in the upper, middle and lower graph respectively. All the TDCSs are in atomic units.

In the three cases, the DW-MC model predicts a lower TDCS amplitude especially in the recoil region. On the other hand, the binary region shape is similar in both TDCSs, with almost the same amplitude for the lower momentum transfer case. The decreasing recoil intensity with both models, especially DW-MC, as we approach the Bethe ridge can be explained by the decrease of backscattering of the ejected electron from the recoil ion which role in the ionization is less and less important.

When these TDCSs are normalized at the same point and compared with the relative experimental TDCSs from [38] (figure 6), they both seem to be in reasonable agreement with the experiment. In the two higher momentum transfer cases, the DW-MC model slightly underestimates the recoil amplitude relatively to the binary amplitude. Both models succeed in describing the shape of the binary region in the three cases. The experimental and theoretical TDCSs exhibit a single binary lobe in the top panels while in the lower panel a double binary peak is observed. This evolution of the single binary peak structure to a double binary peak as the kinematics approach the Bethe ridge was investigated in details for the ionization of the methane $1 \mathrm{t}_{2}$ state of dominant $p$-character in [42]. 


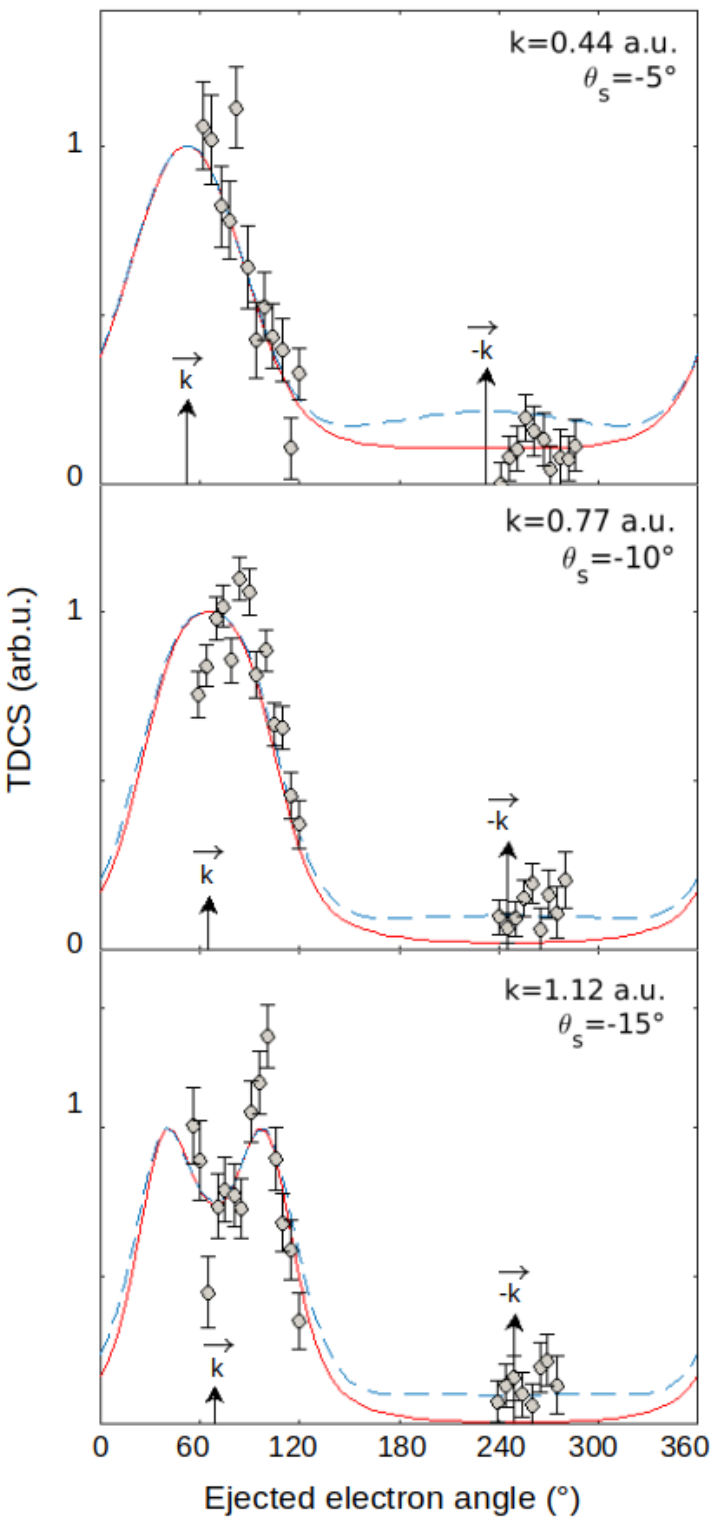

Figure 6: Total triple differential cross sections for the ionization of the HOMO $\left(4 \mathrm{a}^{\prime \prime}\right)$ and $\mathrm{HOMO}^{-1}\left(3 \mathrm{a}^{\prime \prime}\right)$ of phenol using the DW (dashed lines) and DW-MC (solid lines) models compared to experimental TDCSs from [38] (circles with error bars). The kinematic conditions are the same as in figure 5. All the TDCSs are in arbitrary units.

We also note that, in the middle panel of figure $6\left(\theta_{s}=-10^{\circ}\right)$, the experimental binary peak is shifted to the right with respect to the momentum transfer direction. This could be due to post collision interaction (PCI) effects that are beyond the FBA and therefore are not included in both the DW and DW-MC methods. To improve PCI inclusion in our models, we add the Gamow factor [20], as a way to account for the repulsion between the scattered and ejected electrons. In figure 7, we only show the difference between the DW-MC TDCSs and the DW-MC-Gamow TDCSs for a better visual representation of the effect of adding the Gamow factor. 


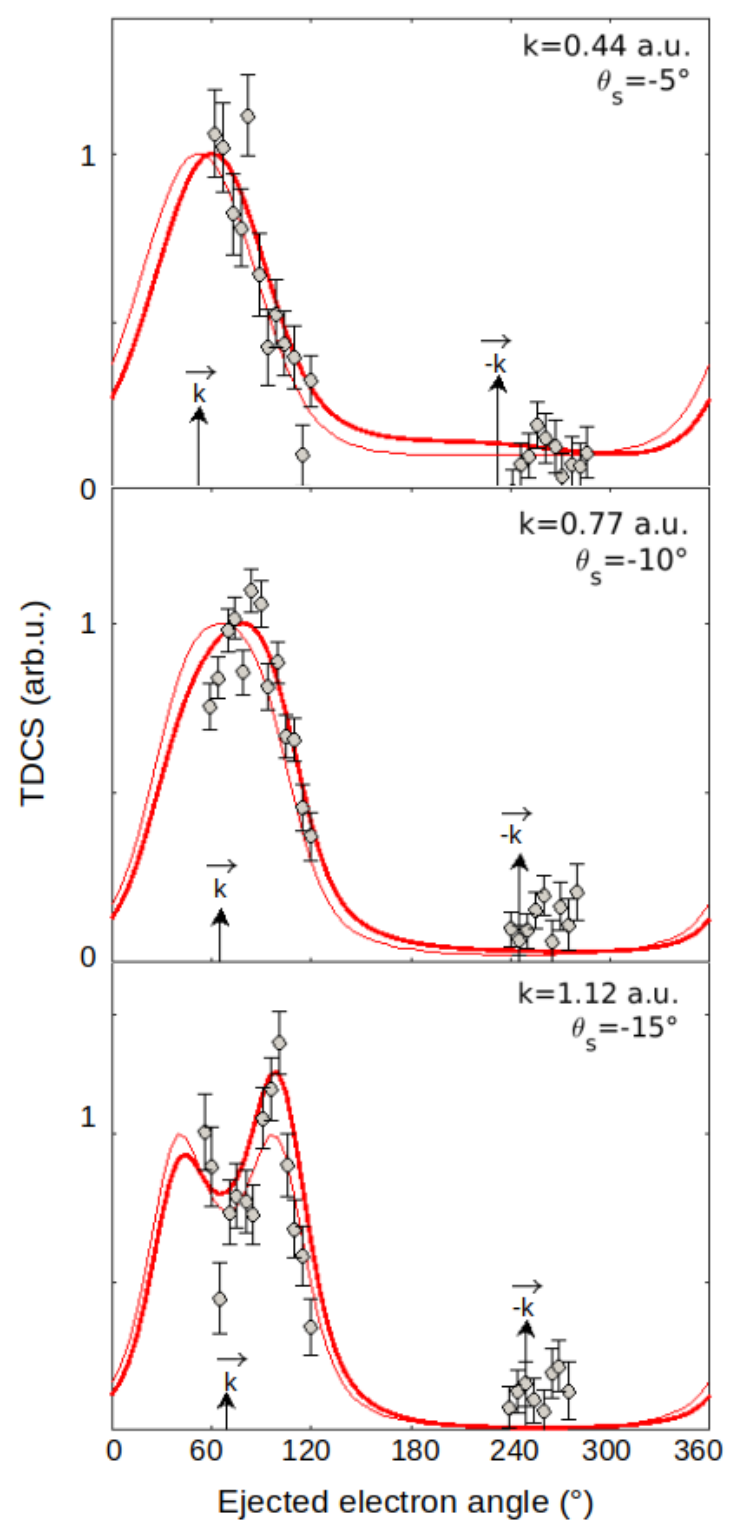

Figure 7: Total triple differential cross sections for the ionization of the HOMO $\left(4 \mathrm{a}^{\prime \prime}\right)$ and HOMO ${ }^{-1}\left(3 \mathrm{a}^{\prime \prime}\right)$ of phenol using the DWMC method with the Gamow factor (thick solid lines) and without the Gamow factor (light solid lines) compared to experimental (circles with error bars) TDCSs from [38]. The kinematic conditions are the same as in figure 5. All the TDCSs are in arbitrary units.

As a result of adding the Gamow factor, the symmetry of the TDCS with respect to the directions of the momentum transfer, which is an imprint of the FBA, is altered. The angular position of the binary peak is improved with the DWMC-Gamow method especially in the $\theta_{s}=-10^{\circ}$ case where the experimental data also exhibit this shift. In the Bethe ridge where PCI effects are the strongest, the Gamow factor clearly improves the results of the DW-MC model by better describing the amplitude difference between the maximum of the second binary peak and the minimum between the two peaks.

We finally compare in figure 8 the results from our four models to the experimental data. We also show M3DW results from [38]. In addition to the experimental TDCSs, Da Silva and co-workers also presented theoretical TDCSs calculated with four different models all of which using the OAMO approximation. The theories that they used all display similar patterns. For that reason and for representation clarity, we only compare to their M3DW results. We note that in the $\theta_{s}=-10^{\circ}$ case, their Gamow factor model slightly improves the results by shifting the amplitude of the second binary peak observed in the M3DW TDCS shown in the dotted curve in the middle panel of figure 8. As can be seen in this figure, their theoretical results are in good agreement with the experimental data in the first case but large discrepancies are observed in the higher scattering angle conditions. They attribute these differences to a failure of the OAMO approximation in correctly describing asymmetric $p$-like orbitals and suggest that the use of the proper average method would improve 
the results but was not possible because of the heaviness of computing. Our results show that it does indeed drastically improve the results as we have better agreement with the experimental data using our four models with the PA approach, in comparison with the much more sophisticated M3DW method but using the OAMO approximation.

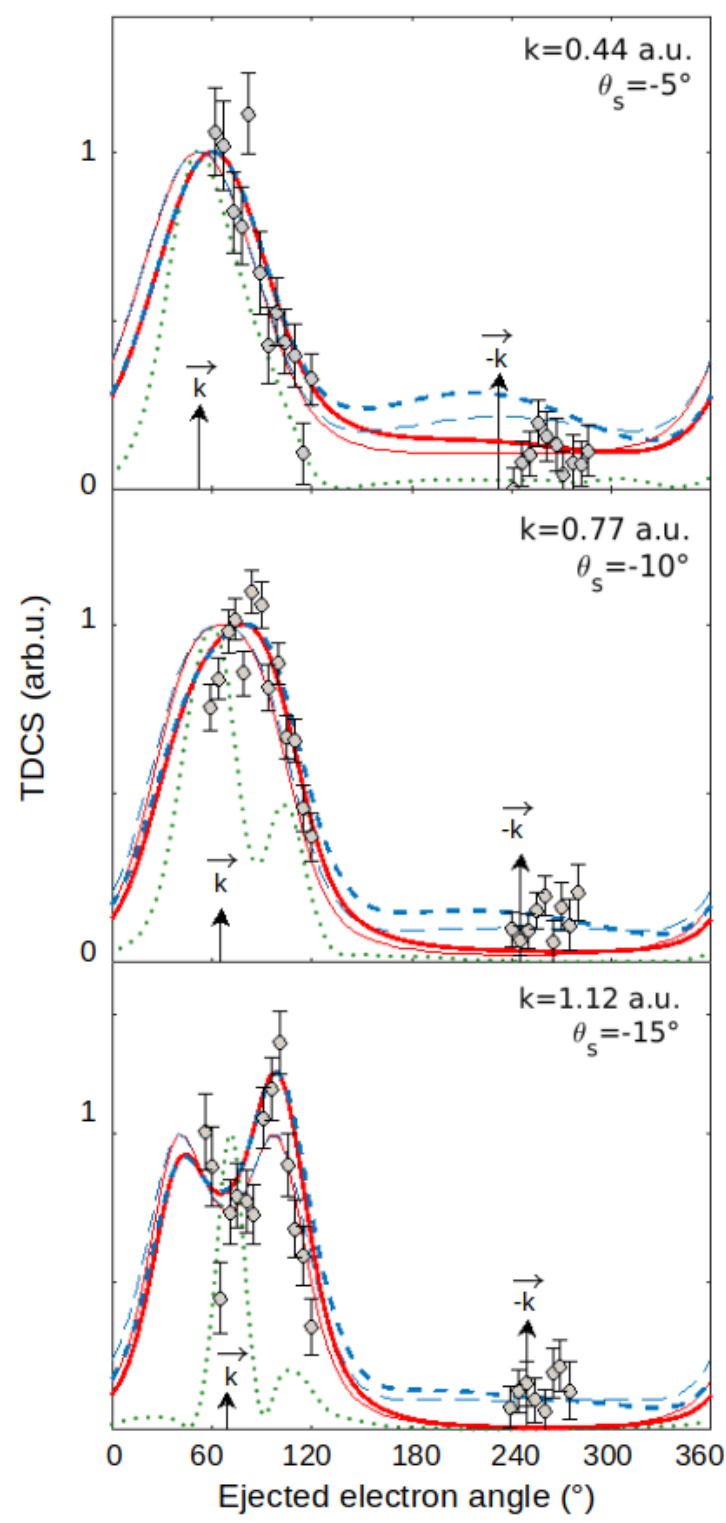

Figure 8: Total triple differential cross sections for the ionization of the HOMO $\left(4 \mathrm{a}^{\prime \prime}\right)$ and HOMO ${ }^{-1}\left(3 \mathrm{a}^{\prime \prime}\right)$ of phenol using the DW (light dashed lines), DW-MC (light solid lines), DW-Gamow (thick dashed lines) and DW-MC-Gamow (thick solid lines) methods, compared to experimental (circles with error bars) and M3DW (dotted lines) TDCSs from [38]. The kinematic conditions are the same as in figure 5. All the TDCSs are in arbitrary units.

\section{Conclusion}

To conclude, we provide in this work theoretical TDCSs for the ionization of the highest (4a") and next-highest $\left(3 \mathrm{a}^{\prime \prime}\right)$ molecular orbitals of phenol by single electron impact. DWBA calculations are performed for incident and ejected electron energies of respectively 250 and $20 \mathrm{eV}$ and for three scattering angles $\left(\theta_{s}=-5^{\circ},-10^{\circ}\right.$ and $\left.-15^{\circ}\right)$ in a coplanar asymmetric geometry. The molecular wave functions of the two orbitals are computed with Gaussian at the RHF level of theory with the $6-31 \mathrm{G}$ basis set. These wave functions are written in a single-center form where the atomic components of these wave functions are expressed relatively to the same geometrical origin. For a valid comparison with the experimental data, a proper average of the TDCS is performed.

Four different models are used in order to investigate the importance of considering the localization of the atoms 
and including the final state electron-electron repulsion in the treatment of the ionization. In these models, the ejected electron is described by a distorted wave calculated as a solution of Schrödinger equation with an angular averaged isotropic distortion potential. In the DW model, the potential seen by the incident electron approaching the target potential does not take into account the localisation of the atoms in the molecule. The DW-MC model is an upgrade of the DW method; it fully includes the nuclear term in the incident electron potential by using the position of the atoms. Moreover, we include the repulsion of the outgoing electrons through the Gamow factor in the DW-Gamow and the DW-MC-Gamow models.

The TDCSs calculated with these four models are in overall good agreement with the experimental data. All models are able to predict the single/double peak structure of the binary region. Just like the experimental patterns, the calculated TDCSs exhibit a single binary peak for the lowest scattering angle, then this peak becomes larger for the higher scattering angle until it splits into two peaks at the highest scattering angle. This evolution of the binary lobe shape as the kinematics approach the Bethe ridge is consistent with the dominant $p$-character of the two orbitals. The DW and DW-MC methods give similar results in the binary region with a slight tightening of the binary lobe with DW-MC as the scattering angle increases. A much more pronounced difference is observed in the recoil region where approximating the potential term that depends on the atoms positions to 1 overestimates the relative recoil amplitude. The influence of including the scattered and ejected electron repulsion is more clear; the binary lobe is pushed away from the momentum transfer direction, improving the angular positions of the binary peaks. In the highest momentum transfer case, the DW-Gamow and DW-MC-Gamow models predict a higher amplitude for the second binary peak which is also more compatible with the experimental data. Hence, the DW-Gamow and DW-MC-Gamow models give the best agreement with the experimental data. We also compare our results to M3DW calculations, performed with the OAMO approximation, and show that much better results are obtained by performing the proper average method.

For relatively complex molecules like phenol, approximations are often crucial to overcome the heaviness of computing. In this work we show that with our methodology we can overcome these challenges and calculate TDCSs for such molecules without having to compromise the validity of the theory. Using accurate molecular wave functions, a proper average of the molecular orientation, including the interaction of the ejected electron with the ionized target and the repulsion between the outgoing electrons, we calculate the TDCSs for a relatively complex molecule at low computational cost. This is made possible especially with the single-center wave function method that greatly reduces the computing time. Therefore our methodology is particularly adapted for complex molecular targets for which little experimental data exist. We strongly encourage more experiments providing TDCSs for the ionization of such molecules.

\section{Acknowledgments}

This work was supported by the CNRS mission for interdisciplinarity (INFINITI proposal, CASIMIR project).

\section{References}

[1] Nikitaki Z et al. 2016 Free Radical Res. 50 S64-S78

[2] Meylan S, Incerti S, Karamitros M, Tang N, Bueno M, Clairand I and Villagrasa C 2017 Sci. Rep. 711923

[3] Sanche L 2005 Eur. Phys. J. D 35 367-390

[4] Alizadeh E, Orlando T M and Sanche L 2015 Annu. Rev. Phys. Chem. 66 379-398

[5] Dal Cappello C, Hervieux P A, Charpentier I and Ruiz-Lopez M F 2008 Phys. Rev. A 78042702

[6] Dal Cappello C, Charpentier I, Houamer S, Hervieux P A, Ruiz-Lopez M F, Mansouri A and Roy A C 2012 J. Phys. B: At. Mol. Opt. Phys. 45175205

[7] Dal Cappello C, Rezkallah Z, Houamer S, Charpentier I, Roy A C, Hervieux P A and Ruiz-Lopez M F 2013 Eur. Phys. J. D 67117

[8] Mouawad L, Hervieux P A, Dal Cappello C, Pansanel J, Osman A, Khalil M and El Bitar Z 2017 J. Phys. B: At. Mol. Opt. Phys. 50215204

[9] Mouawad L, Hervieux P A, Dal Cappello C, Pansanel J, Robert V and El Bitar Z 2018 J. Phys. B: At. Mol. Opt. Phys. 51175201

[10] Mouawad L, Hervieux P A, Dal Cappello C, Pansanel J, Robert V and El Bitar Z 2019 Eur. Phys. J. D 7376

[11] Colyer C J, Bellm S M, Lohmann B, Hanne G F, Al-Hagan O, Madison D H and Ning C G 2010 J. Chem. Phys. 133124302

[12] Jones D B, Builth-Williams J D, Bellm S M, Chiari L, Chaluvadi H, Madison D H, Ning C G, Lohmann B, Ingolfsson O and Brunger M J 2013 Chem. Phys. Lett. 572 32-37 
[13] Builth-Williams J D, Bellm S M, Jones D B, Chaluvadi H, Madison D H, Ning C G, Lohmann B and Brunger M J 2012 J. Chem. Phys. 136024304

[14] Alwan O, Chuluunbaatar O, Assfeld X, Naja A and Joulakian B B 2014 J. Phys. B: At. Mol. Opt. Phys. 47225201

[15] Houamer S, Chinoune M and Dal Cappello C 2017 Eur. Phys. J. D 7117

[16] Gao J, Peacher J L and Madison D H 2005 J. Chem. Phys. 123204302

[17] Colyer C J, Stevenson M A, Al-Hagan O, Madison D H, Ning C G and Lohmann B 2009 J. Phys. B: At. Mol. Opt. Phys. 42235207

[18] Jones D B, Ali E, Ning C G, Colgan J, Ingólfsson O, Madison D H and Brunger M J 2016 J. Chem. Phys. 145 164306

[19] Jones D B et al. 2015 J. Chem. Phys. 143184310

[20] Ward S J and Macek J H 1994 Phys. Rev. A 49 1049-1056

[21] Brauner M, Briggs J S and Klar H 1989 J. Phys. B: At. Mol. Opt. Phys. 22 2265-2287

[22] Gao J, Madison D H and Peacher J L 2006 J. Phys. B: At. Mol. Opt. Phys. 391275

[23] Gao J, Madison D H and Peacher J L 2005 J. Chem. Phys. 123204314

[24] Nixon K L, Murray A J, Chaluvadi H, Ning C G and Madison D H 2011 J. Chem. Phys. 134174304

[25] Chaluvadi H, Ning C G and Madison D H 2014 Phys. Rev. A 89062712

[26] Ali E, Nixon K L, Murray A J, Ning C G, Colgan J and Madison D H 2015 Phys. Rev. A 92042711

[27] Zhang S B, Li X Y, Wang J G, Qu Y Z and Chen X 2014 Phys. Rev. A 89052711

[28] Gong M, Li X, Zhang S B, Liu L, Wu Y, Wang J, Qu Y and Chen X 2017 Phys. Rev. A 96042703

[29] Gong M, Li X, Zhang S B and Chen X 2018 J. Phys. B: At. Mol. Opt. Phys. 51094003

[30] Xu X, Gong M, Li X, Zhang S B and Chen X 2018 J. Chem. Phys. 148244104

[31] Houamer S, Dal Cappello C, Charpentier I, Hervieux P A and Roy A C 2012 Phys. Rev. A 86026701

[32] Chikhalkar S, Jerajani H and Madke B 2013 Int. J. Trichology 5179

[33] Cheng H R and Jiang N 2006 Biotechnol. Lett. 28 55-59

[34] Neves R F C et al. 2015 J. Chem. Phys. 142194302

[35] Neves R F C, Jones D B, Lopes M C A, Blanco F, García G, Ratnavelu K and Brunger M J 2015 J. Chem. Phys. 142 05B609_1

[36] Neves R F C et al. 2015 J. Chem. Phys. 142104305

[37] Da Costa R F et al. 2015 J. Chem. Phys. 142104304

[38] Da Silva G B, Neves R F C, Chiari L, Jones D B, Ali E, Madison D H, Ning C G, Nixon K L, Lopes M C A and Brunger M J 2014 J. Chem. Phys. 141124307

[39] Frisch M J et al. 2016 Gaussian Inc. Wallingford CT

[40] Kaufmann K and Baumeister W 1989 J. Phys. B: At. Mol. Opt. Phys. 221

[41] Khelladi M F, Mansouri A, Dal Cappello C, Charpentier I, Hervieux P A, Ruiz-Lopez M F and Roy A C $2016 J$. Phys. B: At. Mol. Opt. Phys. 49225201

[42] Ali E, Granados C, Sakaamini A, Harvey M, Ancarani L U, Murray A J, Dogan M, Ning C G, Colgan J and Madison D H 2019 J. Chem. Phys. 150194302 